\title{
LEOPOLDO NACHBIN, UM EXCELENTE MATEMÁTICO BRASILEIRO DO SÉCULO XX
}

\author{
Clóvis Pereira \\ Universidade Federal do Paraná - UFPR - Brasil
}

(aceito para publicação em outubro de 2017)

\begin{abstract}
Resumo
Neste artigo homenageamos o matemático brasileiro, Leopoldo Nachbin, que viveu no século XX. No texto abordamos, de modo sumário, além das mais relevantes pesquisas científicas do homenageado, sua decisiva contribuição para a criação de normas administrativas legais que induzissem a formação de recursos humanos qualificados em matemática. Como ideia subjacente, desejamos contribuir, de modo modesto, para a recuperação de uma parte da memória do saber nacional, isto é, a história da matemática no Brasil. Lamentamos profundamente o fato da não existência no país, de um amplo projeto que aborde, de modo sério, a recuperação e conservação do patrimônio matemático brasileiro.
\end{abstract}

Palavras-chave: Leopoldo Nachbin; matemática no Brasil; análise matemática.

\section{[LEOPOLDO NACHBIN, AN EXCELLENT BRAZILIAN MATHEMATICIAN OF THE 20TH CENTURY]}

\begin{abstract}
In this article we pay homage to the Brazilian mathematician, Leopoldo Nachbin, who lived in the 20th century. In the text we approach, in a summary way, besides the most relevant scientific researches of the honoree, his decisive contribution to the creation of legal administrative rules that would induce the formation of qualified human resources in mathematics. As an underlying idea, we wish to contribute, modestly, to the recovery of a part of the memory of national knowledge, that is, the history of mathematics in Brazil. We deeply regret the fact that the country does not have an extensive project that seriously addresses the recovery and conservation of Brazilian mathematical heritage.
\end{abstract}

Keywords: Leopoldo Nachbin; Mathematics in Brazil; Math analysis. 


\section{Introdução}

O objetivo deste artigo é escrever, na forma de uma visão panorâmica, sobre o mais importante matemático brasileiro do século XX, Leopoldo Nachbin, e de modo secundário contribuir para a recuperação da memória do saber matemático nacional ${ }^{1}$. Portanto, o foco de nosso artigo será um cientista que contribuiu, não só com suas pesquisas, publicações, ensino, palestras, orientações e livros, mas também influenciando na parte administrativa, para a criação e formação de instituições de ensino superior, e para a formação do ambiente matemático brasileiro; em especial para a criação e consolidação de linhas de pesquisa em algumas especialidades da Análise Matemática.

Sabe-se que a Análise Matemática clássica foi introduzida no Brasil por Theodoro A. Ramos quando da defesa de sua tese de doutorado, em 25 de junho de 1918, na Escola Politécnica do Rio de Janeiro e intitulada Sobre as Funções de Variáveis Reais, (SILVA, 2003, p. 124 - 130). Posteriormente, com a criação, em especial na Europa, de novas subáreas da Análise, elas foram sendo paulatinamente introduzidas no Brasil, em particular pelo matemático aqui homenageado.

As atividades acadêmicas dos matemáticos brasileiros que nos antecederam merecem melhores divulgações e conhecimentos por parte da comunidade universitária do país. O cientista que homenageamos neste artigo participou do período de efervescência da matemática brasileira, trabalhando no silêncio de seu gabinete, e travando alguns combates silenciosos, e outros não silenciosos, em reuniões científicas, que exigiram qualidades incomuns.

\section{Leopoldo Nachbin}

Leopoldo Nachbin foi um especialista em Análise Matemática e trabalhou, no Brasil, em instituições sediadas na cidade do Rio de Janeiro e em Brasília. No exterior esteve em diversas universidades. Em 1967 foi-lhe oferecida uma posição acadêmica permanente na University of Rochester, USA. Ao aceitar ele ganhou uma George Eastman Professorship, posição que foi criada especialmente para L. Nachbin, e que ele conciliou com suas atividades na UFRJ. Foi o primeiro matemático brasileiro, ainda muito jovem, que trabalhou de modo decisivo para a consolidação, desenvolvimento e estímulo à pesquisa científica em matemática no Brasil. No início dos anos de 1940, em plena $2^{\mathrm{a}}$ Guerra Mundial, quando Leopoldo Nachbin estava iniciando sua carreira científica, as condições de trabalho nas poucas universidades públicas existentes do país eram desfavoráveis para atrair jovens talentosos para seus quadros.

Mesmo sob condições conjunturais desfavoráveis e muitas delas inexistentes, o jovem Leopoldo Nachbin, além de suas pesquisas em matemática, e com visão estratégica

\footnotetext{
${ }^{1}$ Lamentamos o fato de que não existe atualmente um amplo projeto, estimulado ou não pela SBHMat, para Recuperar e Conservar o Patrimônio Matemático Brasileiro. Sabe-se que a FINEP aprovou, em 25 de maio de 2017, apoio financeiro à Academia Nacional de Medicina (ANM) para desenvolver o projeto "Centro da Memória Médica". Este aporte será utilizado para a criação do centro de memórias da instituição, que abrigará os acervos do museu, da biblioteca e do arquivo da Academia Nacional de Medicina.
} 
de futuro, trabalhou de modo decisivo e continuado ao longo de sua vida, para criar no Brasil, incentivos à pesquisa científica. Após a $2^{\text {a }}$ Guerra Mundial, L. Nachbin foi o cientista mais marcante na comunidade acadêmica do Brasil, na organização dos estudos e da pesquisa científica em matemática. Nesta época ele já desfrutava de grande prestígio junto a comunidade matemática internacional.

Nos anos de 1940, L. Nachbin que estava interessado em Teoria dos Espaços Vetorais Topológicos, teoria que surgiu em 1944 após o matemático francês L. Schwartz sistematizar a Teoria de Distribuições, publicou artigos contendo resultados pioneiros em Espaços Vetoriais Topológicos. Em Teoria de Distribuições ele publicou resultados contendo condições necessárias e suficientes para que um espaço de funções contínuas seja bornológico. Ainda nos anos de 1940 L. Nachbin obteve um relevante resultado em Topologia. Ele e o matemático E. Hewitt obtiveram, de modo independente, o resultado que está na literatura matemática como Espaços Saturados ou Espaços Repletos. Estes espaços são conhecidos na literatura matemática por Espaços de Hewitt-Nachbin (WEIR, 1975). Esta classe de espaços foi extensivamente estudada pela comunicade matemática internacional. Nos anos de 1970, L. Nachbin publicou uma nota intitulada Sur les espaces vectoriels topologiques d'applications continues, na qual ele aborda os Espaços de HewittNachbin (NACHBIN, 1970).

Lembramos que L. Nachbin foi um dos fundadores em 1946, e um dos pesquisadores do Núcleo Técnico Científico de Matemática - NTCM da Fundação Getúlio Vargas -FGV, órgão que foi chefiado por Lélio Gama. O NTCM tinha por objetivo coordenar a pesquisa científica em matemática, então iniciando no Brasil, e manter uma ampla comunicação com centros de matemática sediados na América Latina, nos Estados Unidos da América, em Portugal, na Itália e na França. O NTCM teve vida efêmera. Porém criou a importante Revista Summa Brasiliensis Mathematicae, que existiu por mais de 15 anos.

L. Nachbin foi um dos fundadores, em 1948, do Centro Brasileiro de Pesquisas Físicas-CBPF, e um de seus pesquisadores. Também foi um dos fundadores, em 1951, um dos organizadores e um dos pesquisadores do Instituto de Matemática Pura e Aplicada IMPA. Nesta instituição ele e Luis Adauto Medeiros, iniciaram a organização de sua biblioteca.

No início dos anos de 1940, Leopoldo Nachbin, graduado em engenharia civil pela Escola Nacional de Engenharia - ENE da Universidade do Brasil - UB, atual UFRJ, soube aproveitar o ambiente de pesquisa científica em matemática que estava se formando no Departamento de Matemática da Faculdade Nacional de Filosofia -FNFi da UB, com a contratação dos matemáticos italianos G. Mammana (analista) e L. Sobrero (físico matemático) como professores visitantes, e posteriormente, em 1945, com a contratação do matemático português Antonio A. Monteiro, que o incentivou aos estudos de: conjuntos ordenados, reticulados e álgebras de Boole, assuntos atuais para época.

Com o desejo de seguir carreira acadêmica, fazer pesquisa científica em matemática, ministrar aulas, e estimulado especialmente por Antonio Monteiro que o incentivou aos estudos das áreas acima citadas, dentre outras, e porque existia nessa época, segunda metade dos anos de 1940, um teorema de autoria do matemático Marshall Stone para uma álgebra de Boole, explicitando que "um ideal é maximal se, e somente se, ele for 
primo", é que Antonio Monteiro sugeriu a Leopoldo Nachbin a solução de uma questão recíproca para o teorema de Marshall Stone.

Assim, em 1947, Leopoldo Nachbin demostrou no artigo intitulado Une propriété charactéristique des algèbres booleiènnes (NACHBIN, 1947), que um reticulado distributivo contendo primeiro e último elementos é uma álgebra de Boole se, e somente se, todo ideal primo for maximal. Ainda sob a influência científica de Antonio Monteiro, L. Nachbin publicou outros artigos relacionados aos assuntos de estudos sugeridos pelo matemático português (HORVÁTH, 1986).

Em 1948, Leopoldo Nachbin defendeu e foi aprovado, na FNFi da UB, sua tese de livre-docente em Análise, intitulada Combinações de Topologias Metrizáveis e Pseudo Metrizáveis”, assunto muito atual para a Matemática dos anos de 1940. Nesta época, repetimos, a Topologia era uma importante área de pesquisa científica na Europa e nos Estados Unidos da América; na University of Chicago o matemático E. Spanier era, nesta época, um dos destacados especialistas da área.

Lembramos que o concurso público para obtenção do título de livre-docente, não tinha, e não tem nos dias atuais, fins empregatícios com a intituição onde era e é realizado, e concedia e concede ao aprovado o título de livre-docente, tão importante quanto o de doutor $^{2}$. Ainda em 1948, L. Nachbin ganhou uma bolsa de estudos, de um ano, do Departamento de Estado Americano, e em 1949 ganhou outra bolsa de estudos, de um ano, da Fundação Guggenheim, ambas para realizar estágio de pós-doutorado na University of Chicago, USA, onde permaneceu por dois anos. Â época e muitos anos depois, esta instituição era e continuou sendo, um excelente centro de estudos e pesquisa em matemática dos Estados Unidos da América.. Em 1947 e início de 1948 o matemático Marshall Stone, da University of Chicago, esteve como professor visitante no Departamento de Matemática da FNFi da UB. Em 1948 ele assumiu a chefia do Departamento de Matemática da University of Chicago.

De modo simultâneo a suas atividades de ensino e pesquisa, L. Nachbin muito contribuiu com atividades em prol da criação de condições de apoio à pesquisa científica no Brasil. Sua luta foi intensa para serem criadas as estruturas administrativas legais que propiciassem apoio à formação de recursos humanos qualificados em matemática, e também a criação de alguns poucos centros de excelência em matemática no Brasil (NACHBIN, 1956; 1960; 1980). Seu incentivo à ideia do Prof. Dr. Chaim S. Hönig, da USP, para a criação do Colóquio Brasileiro de Matemática foi decisivo. Ele também apoiou, nos anos de 1950, a ideia e o trabalho do Prof. Luiz Freire, físico teórico, para criar

\footnotetext{
2 A livre-docência é um título concedido por uma universidade por meio de concurso aberto. A partir de 11 de setembro de 1976, apenas para portadores do título de doutor. Anteriormente, a livre-docência era aberta a qualquer professor da universidade com a graduação. A introdução da livre-docência no Brasil deve-se à Lei Orgânica do Ensino Superior e do Fundamental, a que se refere o Decreto n ${ }^{\circ} 8.659$, de 5 de abril de 1911, mais conhecida como Reforma Rivadávia Corrêa, por ter sido ele o ministro que referendou o decreto.

O concurso de livre-docência é aberto por edital e o candidato inscrito deverá, além de submeter-se a uma prova escrita e a uma prova didática, deve apresentar uma tese sobre um tema acadêmico e defendê-la perante uma banca examinadora. A livre-docência é regulada pelas Leis $\mathrm{n}^{\circ}$. 5.802/72 e $\mathrm{n}^{\circ}$. 6.096/74, pelo Decreto 76.119/75 e pelo Parecer 826/78 do CFE.
} 
um bom centro de ensino e pesquisa em Matemática na Universidade de Recife, atual UFPE. Este centro marcou o início do atual Departamento de Matemática da UFPE.

Durante a realização do $6^{\circ}$ Colóquio Brasileiro de Matemática, no período de 2 a 22 de julho de 1967, na cidade de Poços de Caldas - MG, evento que teve como coordenador o Prof. Dr. Luis Adauto Medeiros, IM/UFRJ, foi planejada, durante quatros reuniões realizadas com a participação de matemáticos brasileiros e de alguns estrangeiros, a criação da $1^{a}$ Escola Latino Americana de Matemática - ELAM.

Este evento científico foi idealizado por Heitor Gorgolino de Souza, da OEA, e por Leopoldo Nachbin. A ELAM passou a ser realizada sob a responsabilidade da OEA. O que se seguiu à primeira reunião, para a criação da ELAM, gerou um péssimo ambiente acadêmico para o desenvolvimento das pretensões de trabalho de Leopoldo Nachbin e sua equipe, no IMPA, ver (MEDEIROS, 2010, p. 30-31). Segundo (MEDEIROS, 2010, p. 30), "Foi uma surpresa observarmos o surgimento de divergências entre alguns colegas do IMPA com Leopoldo Nachbin, o que criou um clima tenso, difícil".

Como é do conhecimento da comunidade matemática brasileira, talvez não dos mais jovens, poucos anos depois desse incidente L. Nachbin foi afastado de modo abrupto do IMPA. Este fato, segundo relatou o Prof. Dr. Luis Adauto Medeiros, abalou L. Nachbin emocionalmente.

L. Nachbin publicou significativos trabalhos de pesquisa científica em várias revistas de circulação internacional nas seguintes linhas: Sistema Ordenados; Topologia; Espaços Vetoriais Topológicos; Teoria de Aproximação; Análise Harmônica e Holomorfia em Dimensão Infinita. Ele orientou 21 teses de doutorado no Brasil e no exterior. Mas sua descendência matemática contando-se se ele foi orientador 1 , ou orientador 2, chega a 148 alunos. Além de profundo conhecedor de seu campo de pesquisa e ensino, L. Nachbin possuía o dom de bem transmitir seus conhecimentos. Ele foi um expositor de qualidade excepcional.

Em 1949, quando estava nos Estados Unidos da América, e trabalhando em teoria de aproximação, Leopoldo Nachbin publicou o artigo intitulado Sur les algèbres denses de fonctions différentiables sur une variété, contendo uma caracterização de subálgebras densas de funções diferenciáveis, ver (NACHBIN, 1949). Este foi um de seus artigos mais relevantes. Este resultado por ele obtido em funções diferenciáveis é um análogo do conhecido teorema de Weirstrass - Stone, que é uma generalização feita por Marshall Stone (STONE, 1937), do teorema de aproximação de Weierstrass. Este teorema é conhecido dos alunos de um bom curso de graduação em matemática; ele está na literatura matemática desde 1885. O teorema de Weierstrass informa o seguinte: "Toda função contínua $f$ no intervalo fechado $[\mathrm{a}, \mathrm{b}]$ de $\mathbb{R}(f:[\mathrm{a}, \mathrm{b}] \rightarrow \mathbb{R})$, é limite uniforme de uma sucessão de polinômios".

No ano de 1950 a FNFi da UB abriu concurso para o cargo de professor catedrático da cadeira de Análise Matemática e Análise Superior, do Departamento de Matemática. L. Nachbin se inscreveu, pois ele estava habilitado por ser livre-docente em Análise Matemática, e apresentou a tese intitulada Topologia e Ordem, trabalho que ele escreveu quando estava na University of Chicago. Este concurso foi realizsado 22 anos depois para o cargo de professor titular. Como sabemos o cargo de professor catedrático foi extinto quando da reforma da universidade brasileira realizada pelo governo federal no final 
dos anos de 1960 e início dos anos de 1970. Para uma estória sobre este concurso, (SILVA, 2013, p. 230-231; MEDEIROS, 2010, p. 26,27,28).

No ano de 1948 L. Nachbin se interessou pela união de duas estruturas com as quais jé estava trabalhando algum tempo. Como efeito desse intersse ele escreveu o trabalho intitulado Topologia e Ordem que o apresentou, como tese, para o concurso acima citado. Em síntese, este trabalho aborda uma caracterização dos espaços topológicos normalmente pré-ordenados que obtem-se por meio de uma generalização do teorema de separação, resultado em Topologia Geral que ele obteve por meio de trabalhos de Pavel Urysohn e André Weil.

Em Topologia e Ordem, sua tese para o concurso, L. Nachbin introduziu os conceitos de: Espaço Topológico Normalmente Ordenado, Espaço Compacto Ordenado e Espaço Uniforme Ordenado. Ele generalizou a estes espaços os resultados da teoria já obtidos por P. Urysohn e A. Weil. Para detalhes técnicos, (MEDEIROS, 2001, p.19-27).

Em Análise Funcional, nos anos de 1950, L. Nachbin publicou seu famoso resultado na forma de um teorema. Este é um de seus mais relevantes resultados obtido e certamente o que mais foi citado pela comunidade matemática mundial. Trata-se do seguinte: A theorem of the Hahn-Banach type for linear transformações, resultado que generaliza o conhecido teorema de Hahn-Banach, que é encontrado na literatura matemática. Relembramos que este teorema, por exemplo, em sua forma no corpo dos reais, é um resultado fundamental da Análise Funcional, pois ele permite que funcionais lineares definidos em um subespaço de um espaço vetorial $\mathrm{V}$, sejam estendidos a todo o espaço. Para detalhes técnicos sobre os resultados obtidos por L. Nachbin (NACHBIN, 1950).

Em 1953 o matemático francês J. Dieudonné propôs, à comunidade matemática mundial, o seguinte problema: decidir se todo espaço tonelado seria necessariamente bornológico (DIEUDONNÉ, 1953). L. Nachbin resolveu este problema em sua forma negativa (NACHBIN, 1954). Eis o seu teorema para a solução desse problema.

Seja $\mathrm{X}$ um espaço de Hausdorff completamente regular, e consideremos $\mathrm{C}(\mathrm{X})$ munido da topologia compacto-aberta.

1) $\mathrm{C}(\mathrm{X})$ é tonelado se e só se, para cada subconjunto fechado, mas não compacto $\mathrm{A}$ de $X$, existe uma função $f \in C(X)$ que não é limitada sobre $A$;

2)C(X) é bornológico se e só se, $X$ é repleto.

Na demonstração do teorema acima citado, L. Nachbin mostrou que existem espaços que verificam a condição 1), mas que não são repletos, fato que resolve, pela negativa, o problema proposto por J. Dieudonné.

Este problema foi também demonstrado, de modo simultâneo e independente à demonstração de L. Nachbin, pelo matemático T. Shirota (SHIROTA, 1954). O resultado dos dois está na literatura matemática como teorema de Nachbin-Shirota. 
Outro dos mais relevantes resultados obtidos de L. Nachbin está em aproximação ponderada de funções contínuas. O problema de aproximação ponderada de S. Bernstein (BERNSTEIN, 1924), consiste em obter condições para que um peso v seja ponderado. Ao estudar este problema L. Nachbin o generalizou (NACHBIN, 1964; 1965), de modo análogo que M. Stone havia generalizado o problema de aproximação de Weierstrass citado acima.

Ao se estabelecer no IM/UFRJ, após sua saída abrupta do IMPA no início dos anos de 1970, L. Nachbin criou para seus alunos do programa de doutorado, um programa de pesquisas em Análise Complexa em Dimensão Infinita. Desse modo, seus alunos de doutorado no IM/UFRJ passaram a trabalhar em: Espaços de Germes de Aplicações Holomorfas em Espaços Vetoriais Topológicos Localmente Convexos.

Ainda nos anos de 1970, no IM/UFRJ, L. Nachbin estendeu os conceitos de tonelagem e de bornologia para uma teoria análoga, utilizando para isso propriedades de funções Holomorfas.

\section{Considerações Finais}

Em função da vasta atuação acadêmica e do grande número de publicações científicas, 110 publicações, e livros, 15 livros, que foram produzidos por L. Nachbin em matemática, e impossibilitados pelo limite superior, do número de páginas para escrever este artigo, uma condição compreensível, decidimos fazer esta modesta homenagem a L. Nachbin, com foco em alguns de seus mais relevantes trabalhos publicados, e por meio de uma visão panorâmica de suas atividades acadêmicas e administrativas, no período em que esteve entre nós.

As influências científicas exercidas por L. Nachbin não se restringiram apenas à comunidade matemática brasileira. Seus trabalhos de estudos e pesquisas científicas em forma de aulas, orientações, conferências e seminários ministrados no Brasil e no exterior, além de livros publicados no Brasil e no exterior, e artigos publicados em revistas de circulação internacional, influenciaram várias gerações de jovens talentosos em seus estudos e pesquisas em Análise Matemática e em outras subáreas da matemática e também nos estudos e pesquisa em Probabilidade/Estatística e Economia Matemática. Para a relação completa de seus livros e artigos publicados (HORVÁTH, 1984).

Os esforços de L. Nachbin visando à melhoria da qualidade na formação de recursos humanos em matemática para o exercício de atividades docentes nas universidades brasileiras e em universidades do Uruguai, Argentina, Chile, Perú, Colômbia e Venezuela, também foi uma de suas batalhas. Nos anos de 1960 o Diretor do IMPA solicitou-lhe, para fins de estudos em andamento, dados numéricos e qualitativos sobre os aspectos seguintes: 1) número de matemáticos pesquisadores brasileiros ausentes do país em longo prazo; 2) 
motivos determinantes de um tal êxodo. Em carta resposta ao Diretor do IMPA ele apresentou os motivos, segundo sua experiência acadêmica (IMPA, 1965).

Em 1962 L. Nachbin foi conferencista convidado do International Congress of Mathemticians - ICM, que foi realizado em Stockholm, Suécia. Aliás, ele foi o primeiro matemático brasileiro a ser convidado para proferir uma conferência nesse importante evento científico. Dentre os títulos acadêmicos recebidos por L. Nachbin no Brasil, citamos os seguintes. Em 1973 ele recebeu o título de Doutor Honoris Causa da UFPE; em 1989 ele foi agraciado com o título de Professor Honorário da UNICAMP.

L. Nachbin soube aproveitar, por meio de contatos científicos, a presença no Brasil, no período de 1945 a 1954, de importantes matemáticos estrangeiros como professores visitantes da USP e da UB. Dentre estes matemáticos citamos de modo aleatório: A. Monteiro, A. A. Albert, M. H. Stone, Warren Ambrose, O. Zariski, A. Weil, J. Dieudonné, L. Schwartz, A. Grothendieck. Segundo suas palavras, seu mentor foi o matemático francês L. Schwartz, com quem manteve frutífero contato científico. Ele influenciou cientificamente L. Nachbin para estudos e pesquisas em Análise Funcional e em Teoria de Distribuições. Posteriormente, este matemático convidou L. Nachbin para ser professor visitante na Universidade de Paris.

Em 1948 Leopoldo Nachbin foi eleito Membro Associado da Academia Brasileira de Ciências - ABC; em 1950 ele passou a ser Membro Titular da ABC. No período de 1954 a 1961, L. Nachbin foi sucessivamente, Diretor do Setor de Pesquisas Matemáticas do CNPq e Membro do Conselho Deliberativo do CNPq. No período de 1961 a 1970 ele foi Coordenador do Instituto Central de Matemática da UnB.

Nossos agradecimentos ao Prof. Dr. Gervásio G. Bastos, (UFC) por ter sugerido que escrevêssemos este artigo, e por sua leitura.

\section{Referências Bibliográficas}

BERNSTEIN, S. Le probleme de l'aproximation des fonctions continues sur tout l'axe reel et l'une de ses applications. Bull. Soc. Math. France, 52, p. 399-410, 1924.

DIEUDONNÉ, J. Recent developments in the theory of locally convex vector spaces. Bull. Amer. Math. Soc., 59, p. 495-512, 1953.

HORVÁTH, John. The Life and Work of Leopoldo Nachbin. Ed. University of Maryland, 1984.

IMPA. Noticiário Brasileiro de Matemática, nº 19, 20, 21, 1965.

MEDEIROS, Luis A. Trajeto da Matemática no Rio de Janeiro. Ciência e Sociedade, ${ }^{\circ}$ 003/01. Rio de Janeiro: CBPF, 2001. Alfarrábio - I. Rio de Janeiro, 2010.

NACHBIN, Leopoldo. Une propriété charactéristique des algèbres booleiènnes. Portugalae Math. 6, p. 115 - 118, 1947. 
Sur les algèbres denses de fonctions différentiables sur une variété. $C . R$. Acad. Sci. Paris, 228, p. 1549-1551, 1949.

. A theorem of the Hahn-Banach type for linear transformations. Trans. Amer. Math. Soc., 68, p. 28-46, 1950.

Topological vector spaces of continuous functions. Proc. Nat. Acad. Sci., 40, p.471-472, 1954.

. Aspectos do Desenvolvimento Recente da Matemática no Brasil. Anuário da Sociedade Paranaense de Matemática, v. 3, p. 28 - 41, 1956.

$-4,1960$. O Que é o IMPA? Bol. Sociedade Paranaense de Matemática, v. 3, nº 1, p. 1

. Weighted approximation over topological spaces and the Bernstein problem over finite dimensional vector spaces. Topology, 3, Suppl. 1, p. 125-130, 1964.

. Elements of approximation theory. Univ. of Rochester, 1964.

. Weighted approximation for algebras and modules of continuous functions: real and self-adjoint complex cases. Ann. of Math., 2, p. 289-302, 1965.

. Sur les espaces vectoriels topologiques d'applications continues. C. R. Acad. Sci. Paris, 271, p. 596-598, 1970.

. The Influence of Antonio A. Ribeiro Monteiro in the Development of Mathematics in Brazil. Portugalae Mathematica, v. 39, fasc.1 - 4, p. 15 - 17, 1980.

SHIROTA, T. On locally convex vector spaces of continuous functions. Proc. Japan Acad., 30, p. 294-298, 1954.

SILVA, Clóvis Pereira da. A Matemática no Brasil: História de seu desenvolvimento. $3^{\mathrm{a}}$ Edição revista. São Paulo: Edgard Blucher, 2003.

. Início e Consolidação da Pesquisa em Matemática no Brasil, $2^{\text {a }}$ ed. Rio de Janeiro: Ed. Ciência Moderna, 2013.

STONE, Marshall H. Applications of the theory of Boolean rings to general topology. Trans. Amer. Math. Soc. 41, p. 375 - 481, 1937.

WEIR, M. D. Hewitt-Nachbin spaces. Amsterdam: North Holland, Math Studies 17, 1975.

\section{Clóvis Pereira}

Professor aposentado - UFPR - Curitiba - Brasil

E-mail: clovisps@uol.com.br 